\title{
Computation of Substring Probabilities in Stochastic Grammars
}

\author{
Ana L. N. Fred \\ Instituto de Telecomunicações \\ Instituto Superior Técnico \\ IST-Torre Norte, Av. Rovisco Pais, 1049-001 Lisboa, Portugal \\ afred@lx.it.pt
}

\begin{abstract}
The computation of the probability of string generation according to stochastic grammars given only some of the symbols that compose it underlies pattern recognition problems concerning the prediction and/or recognition based on partial observations. This paper presents algorithms for the computation of substring probabilities in stochastic regular languages. Situations covered include prefix, suffix and island probabilities. The computational time complexity of the algorithms is analyzed.
\end{abstract}

\section{Introduction}

The computation of the probability of string generation according to stochastic grammars given only some of the symbols that compose it, underlies some pattern recognition problems such as prediction and recognition of patterns based on partial observations. Examples of this type of problems are illustrated in [2$4]$, in the context of automatic speech understanding, and in [5,6], concerning the prediction of a particular physiological state based on the syntactic analysis of electro-encephalographic signals. Another potential application, in the area of image understanding, is the recognition of partially occluded objects based on their string contour descriptions.

Expressions for the computation of substring probabilities according to stochastic context-free grammars written in Chomsky Normal Form have been proposed [1-3]. In this paper algorithms for the computation of substring probabilities for regular-type languages expressed by stochastic grammars of the form

$$
\sigma \rightarrow F_{i} \quad, \quad F_{i} \rightarrow \alpha \quad, \quad F_{i} \rightarrow \alpha F_{j} \quad, \quad \alpha \in \Sigma^{*} \quad, \quad \sigma, F_{i}, F_{j} \in V_{N}
$$

( $\sigma$ representing the grammar start symbol and $V_{N}, \Sigma$ corresponding to the nonterminal and terminal symbols sets, respectively) are described. This type of grammars arises, for instance, in the process of grammatical inference based on Crespi-Reghizzi's method [7] when structural samples assume the form

$$
[\ldots[d c[f g b[e[c d[a b]]]]
$$

(meaning some sort of temporal alignment of sub-patterns). 
The algorithms presented next explore the particular structure of these grammars, being essentially dynamic programming methods. Situations described include the recognition of fixed length strings (probability of exact recognition section 3.1, highest probability interpretation - section 3.2) and arbitrary length strings (prefix - section 3.3, suffix - section 3.5 and island probabilities - section 3.4). The computational complexity of the methods is analyzed in terms of worst case time complexity. Minor and obvious modifications of the above algorithms enable the computation of probabilities according to the standard form of regular grammars.

\section{Notation and Definitions}

Let $\mathcal{G}=\left(V_{N}, \Sigma, R_{s}, \sigma\right)$ be a stochastic context-free grammar, where $V_{N}$ is the finite set of non-terminal symbols or syntactical categories; $\Sigma$ is the set of terminal symbols (vocabulary); $R_{s}$ is a finite set of productions of the form $p_{i}: A \rightarrow \alpha$, $A \in V_{N}, \alpha \in\left(\Sigma \cup V_{N}\right)^{*}$, the star representing any combination of symbols in the set and $p_{i}$ is the rule probability; and $\sigma \in V_{N}$ is the start symbol. When rules take the particular form $A \rightarrow a B$ or $A \rightarrow a$, with $A, B \in V_{N}$ and $a \in \Sigma$, then the grammar is designated as finite-state or regular.

Along the text the symbols $A, G$ and $H$ will be used to represent non-terminal symbols. The following definitions are also useful:

$w_{1} \ldots w_{n} \stackrel{\text { def }}{=}$ finite sequence of terminal symbols;

$H \stackrel{*}{\Rightarrow} \alpha \stackrel{\text { def }}{=}$ derivations of $\alpha$ from $H$ through the application of an arbitrary number of rules;

$C_{T}(\gamma) \stackrel{\text { def }}{=}$ number of terminal symbols in $\gamma$ (with repetitions);

$C_{N}(\gamma) \stackrel{\text { def }}{=}$ number of non-terminal symbols in $\gamma$ (with repetitions);

$n_{\min (H, G)} \stackrel{\text { def }}{=} \min \left\{n, \stackrel{\max }{\gamma}\left\{C_{T}(\gamma): H \rightarrow \gamma G\right\}\right\}$

The following graphical notation is used to represent derivation trees:

- Arcs are associated with the direct application of rules. For instance, the rule $H \rightarrow w_{1} w_{2} G$ is represented by $\overbrace{w_{1} w_{2}}^{H}$ or $\overbrace{w_{1} w_{2}}^{H} G$

- The triangle represents derivation trees having the top non-terminal symbol as root and leading to the string on the base of the triangle:

$$
H \stackrel{*}{\Rightarrow} w_{1} \ldots w_{n} \Sigma^{*} \equiv \overbrace{w_{1} \ldots w_{n}}^{H} \Sigma^{*}
$$




\section{Algorithms Description}

\subsection{Probability of Derivation of Exact String $-\operatorname{Pr}\left(H \stackrel{*}{\Rightarrow} w_{i} \ldots w_{i+n}\right)$}

Let $\operatorname{Pr}\left(H \stackrel{*}{\Rightarrow} w_{i} \ldots w_{i+n}\right)$ be the probability of all derivation trees having $H$ as root and generating exactly $w_{i} \ldots w_{i+n}$.

According to the type of grammars considered the computation of this probability can be obtained as follows (see figure 1):

$$
\begin{aligned}
& \operatorname{Pr}\left(\sigma \stackrel{*}{\Rightarrow} w_{i} \ldots w_{i+n}\right)=\sum_{G} \operatorname{Pr}(\sigma \rightarrow G) \operatorname{Pr}\left(G \stackrel{*}{\Rightarrow} w_{i} \ldots w_{i+n}\right) \\
& \operatorname{Pr}\left(H \stackrel{*}{\Rightarrow} w_{i} \ldots w_{i+n}\right) \quad, \quad H \neq \sigma \\
& =\operatorname{Pr}\left(H \rightarrow w_{i} \ldots w_{i+n}\right)+ \\
& \quad+\sum_{G} \sum_{k=1}^{n_{\text {min }}(H, G)} \operatorname{Pr}\left(H \rightarrow w_{i} \ldots w_{i+k-1} G\right) \operatorname{Pr}\left(G \stackrel{*}{\Rightarrow} w_{i+k} \ldots w_{i+n}\right)
\end{aligned}
$$
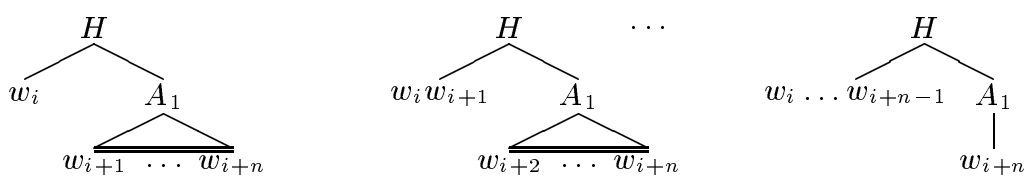

Fig. 1. Terms of the expression 2.

This can be summarized by the iterative algorithm:

1. For all non-terminal symbols $H \in V_{N}-\{\sigma\}$, determine

$$
\operatorname{Pr}\left(H \rightarrow w_{i+n}\right)
$$

2. For $k=1, \ldots n$ and for all non-terminal symbols $H \in V_{N}-\{\sigma\}$, compute:

$$
\begin{aligned}
& \operatorname{Pr}\left(H \stackrel{*}{\Rightarrow} w_{i+n-k} \ldots w_{i+n}\right) \\
& =\operatorname{Pr}\left(H \rightarrow w_{i+n-k} \ldots w_{i+n}\right)+ \\
& \quad+\sum_{G} \sum_{j=1}^{n_{\min (H, G)}} \operatorname{Pr}\left(H \rightarrow w_{i+n-k} \ldots w_{i+n-k+j-1} G\right) \times \\
& \quad \operatorname{Pr}\left(G \stackrel{*}{\Rightarrow} w_{i+n-k+j} \ldots w_{i+n}\right)
\end{aligned}
$$


3.

$$
\operatorname{Pr}\left(\sigma \stackrel{*}{\Rightarrow} w_{i} \ldots w_{i+n}\right)=\sum_{G} \operatorname{Pr}(\sigma \rightarrow G) \operatorname{Pr}\left(G \stackrel{*}{\Rightarrow} w_{i} \ldots w_{i+n}\right)
$$

This corresponds to filling in the matrix of figure 2, column by column, from the right to the left. In this figure, each element corresponds to the probability of all derivation trees for the substring on the top of the column, with root on the non-terminal symbols indicated on the left of the row, i.e. $\operatorname{Pr}\left(H_{i} \stackrel{*}{\Rightarrow} w_{j} \ldots w_{i+n}\right)$.

\begin{tabular}{l|c|c|c|c|} 
& $w_{i} \ldots w_{i+n}$ & $\ldots$ & $w_{i+n-1} \ldots w_{i+n}$ & $w_{i+n}$ \\
\hline$H_{1}$ & & & & $\operatorname{Pr}\left(H_{1} \rightarrow w_{i+n}\right)$ \\
\hline$H_{2}$ & & & $\operatorname{Pr}\left(H_{2} \stackrel{*}{\Rightarrow} w_{i+n-1} w_{i+n}\right)$ & \\
\hline$\vdots$ & & & & \\
\hline$H_{\left|V_{N}\right|}$ & & & & \\
\hline
\end{tabular}

Fig. 2. Array representing the syntactic recognition of strings according to the algorithm.

Notice that the calculations on step 2 are based only on direct rules and access to previously computed probabilities on columns to the right of the position under calculation.

Computational complexity analysis For strings of length $n$, the computational cycle is repeated $n$ times, all non-terminal symbols being considered. Associating to the terms in equation 3

$$
\begin{aligned}
& \text { A: } \operatorname{Pr}\left(H \rightarrow w_{i+n-k} \ldots w_{i+n}\right) \\
& \mathrm{B}: \quad \sum_{G} \sum_{j=1}^{n_{\min (H, G)}} \operatorname{Pr}\left(H \rightarrow w_{i+n-k} \ldots w_{i+n-k+j-1} G\right) \operatorname{Pr}\left(G \stackrel{*}{\Rightarrow} w_{i+n-k+j} \ldots w_{i+n}\right)
\end{aligned}
$$

the worst case time complexity is of the order

$$
\begin{array}{r}
O(\underbrace{\left(\left|V_{N}\right|-1\right) \delta}_{\text {step } 1}+\underbrace{\sum_{k=1}^{n-1}\left(\left|V_{N}\right|-1\right)(\underbrace{\text { computation of A }}_{\alpha}+\underbrace{\text { computation of B }}_{\beta})}_{\text {step 2 }}+\underbrace{\theta}_{\text {step } 3}) \\
=O\left(\left|V_{N}\right| n\right)
\end{array}
$$




\subsection{Maximum Probability of Derivation $-P_{m}\left(H \stackrel{*}{\Rightarrow} w_{i} \ldots w_{i+n}\right)$}

Let $\mathrm{P}_{m}\left(H \stackrel{*}{\Rightarrow} w_{1} \ldots w_{n}\right)$ denote the highest probability over all derivation trees having $H$ as root and producing exactly $w_{1} \ldots w_{n}$ and define the matrix $M_{u}$ as follows:

$$
M_{u}[i, j]=\mathrm{P}_{m}\left(H_{i} \stackrel{*}{\Rightarrow} w_{j} \ldots w_{n}\right) \quad i=1, \ldots,\left|V_{N}\right| \quad j=1, \ldots, n
$$

Observing that

$$
\mathrm{P}_{m}\left(\sigma \stackrel{*}{\Rightarrow} w_{1} \ldots w_{n}\right)=\max _{G}\left\{\mathrm{P}_{m}\left(G \stackrel{*}{\Rightarrow} w_{1} \ldots w_{n}\right)\right\}
$$

and

$$
\begin{aligned}
\mathrm{P}_{m}\left(H \stackrel{*}{\Rightarrow} w_{1} \ldots w_{n}\right) \quad, \quad H \neq \sigma \\
=\max \left\{\operatorname{Pr}\left(H \rightarrow w_{1} \ldots w_{n}\right),\right. \\
\left.\quad \max _{i, G}\left\{\operatorname{Pr}\left(H \rightarrow w_{1} \ldots w_{i} G\right) P_{m}\left(G \stackrel{*}{\Rightarrow} w_{i+1} \ldots w_{n}\right)\right\}\right\}
\end{aligned}
$$

the following algorithm computes the desired probability:

1. For $i=1, \ldots,\left|V_{N}\right| \quad, H_{i} \neq \sigma$

$$
M_{u}[i, n]= \begin{cases}\operatorname{Pr}\left(H_{i} \rightarrow w_{n}\right) & \text { if }\left(H_{i} \rightarrow w_{n}\right) \in R_{s} \\ 0 & \text { otherwise }\end{cases}
$$

2. For $j=n-1, \ldots, 1$ and for $i=1, \ldots,\left|V_{N}\right| \quad, H_{i} \neq \sigma$

$$
\begin{aligned}
& M_{u}[i, j]=\max \left\{\operatorname{Pr}\left(H_{i} \rightarrow w_{j} \ldots w_{n}\right),\right. \\
& \left.\max _{k>j, l}\left\{\operatorname{Pr}\left(H_{i} \rightarrow w_{j} \ldots w_{k} H_{l}\right) M_{u}[l, k+1]\right\}\right\}
\end{aligned}
$$

3. For $i: H_{i}=\sigma$

$$
M_{u}[i, 1]=\max _{j}\left\{\operatorname{Pr}\left(\sigma \rightarrow H_{j}\right) M_{u}[j, 1]\right\}
$$

This algorithm corresponds to the computation of an array similar to the one in figure 2, but where probabilities refer to maximum values of single derivations instead of total probability of derivation when ambiguous grammars are considered.

Based on the similarity between this algorithm and the one developed in section 3.1 it is straightforward to conclude that it runs in $O\left(\left|V_{N}\right| n\right)$ time. 


\subsection{Prefix Probability $-\operatorname{Pr}\left(H \stackrel{*}{\Rightarrow} w_{i} \ldots w_{i+n} \Sigma^{*}\right)$}

The probability of all derivation trees having $H$ as root and generating arbitrary length strings with the prefix $\left.w_{i} \ldots w_{i+n}-\operatorname{Pr}\left(H \stackrel{*}{\Rightarrow} w_{i} \ldots w_{i+n} \Sigma^{*}\right)\right)-$ can be expressed as follows:

$$
\begin{aligned}
& \operatorname{Pr}\left(\sigma \stackrel{*}{\Rightarrow} w_{i} \ldots w_{i+n} \Sigma^{*}\right)=\sum_{G} \operatorname{Pr}(\sigma \rightarrow G) \operatorname{Pr}\left(G \stackrel{*}{\Rightarrow} w_{i} \ldots w_{i+n} \Sigma^{*}\right) \\
& \operatorname{Pr}\left(H \stackrel{*}{\Rightarrow} w_{i} \ldots w_{i+n} \Sigma^{*}\right) \quad, \quad H \neq \sigma \\
&=\operatorname{Pr}\left(H \rightarrow w_{i} \ldots w_{i+n}\right)+ \\
& \quad+\sum_{G} \sum_{k=1}^{n_{\min (H, G)}} \operatorname{Pr}\left(H \rightarrow w_{i} \ldots w_{i+k-1} G\right) \operatorname{Pr}\left(G \stackrel{*}{\Rightarrow} w_{i+k} \ldots w_{i+n} \Sigma^{*}\right)+ \\
& \quad+\sum_{G} \operatorname{Pr}\left(H \rightarrow w_{i} \ldots w_{i+n} G\right)+ \\
& \quad+\sum_{G} \sum_{k \in \mathcal{N}^{+}} \operatorname{Pr}\left(H \rightarrow w_{i} \ldots w_{i+n} v_{1} \ldots v_{k} G\right) \\
&=\operatorname{Pr}\left(H \rightarrow w_{i} \ldots w_{i+n}\right)+ \\
& \quad+\sum_{G}^{n_{\min }(H, G)} \sum_{k=1} \operatorname{Pr}\left(H \rightarrow w_{i} \ldots w_{i+k-1} G\right) \operatorname{Pr}\left(G \stackrel{*}{\Rightarrow} w_{i+k} \ldots w_{i+n} \Sigma^{*}\right)+ \\
& \quad+\sum_{G, k \in \mathcal{N}_{0}^{+}} \operatorname{Pr}\left(H \rightarrow w_{i} \ldots w_{i+n} v_{1} \ldots v_{k} G\right)
\end{aligned}
$$

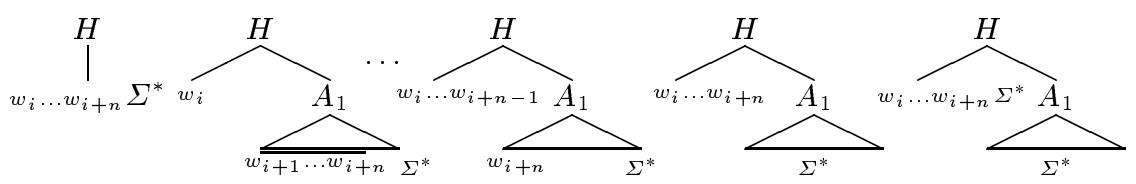

Fig. 3. Terms of the expression 9 .

The previous expression suggest the following iterative algorithm:

1. For all nonterminal symbols $H \in V_{N}-\{\sigma\}$, determine

$$
\operatorname{Pr}\left(H \rightarrow w_{i+n}\right)+\sum_{k \in \mathcal{N}^{+}} \operatorname{Pr}\left(H \rightarrow w_{i+n} v_{1} \ldots v_{k}\right)
$$

2. For $k=1, \ldots, n$ and for all nonterminal symbols $H \in V_{N}-\{\sigma\}$, compute

$$
\operatorname{Pr}\left(H \stackrel{*}{\Rightarrow} w_{i+n-k} \ldots w_{i+n} \Sigma^{*}\right)
$$




$$
\begin{aligned}
& =\operatorname{Pr}\left(H \rightarrow w_{i+n-k} \ldots w_{i+n}\right)+ \\
& +\sum_{G} \sum_{j=1}^{k_{\min (H, G)}} \operatorname{Pr}\left(H \rightarrow w_{i+n-k} \ldots w_{i+n-k+j-1} G\right) \times \\
& \quad \times \operatorname{Pr}\left(G \stackrel{*}{\Rightarrow} w_{i+n-k+j} \ldots w_{i+n} \Sigma^{*}\right)+ \\
& +\sum_{G} \sum_{j \in \mathcal{N}_{0}^{+}} \operatorname{Pr}\left(H \rightarrow w_{i+n-k} \ldots w_{i+n} v_{1} \ldots v_{j} G\right)
\end{aligned}
$$

3.

$$
\operatorname{Pr}\left(\sigma \stackrel{*}{\Rightarrow} w_{i} \ldots w_{i+n} \Sigma^{*}\right)=\sum_{G} \operatorname{Pr}(\sigma \rightarrow G) \operatorname{Pr}\left(G \stackrel{*}{\Rightarrow} w_{i} \ldots w_{i+n} \Sigma^{*}\right)
$$

This algorithm, like the one of section 3.1, corresponds to filling in a parsing matrix similar to the one in figure 2 .

The similarity with the algorithm in section 3.1 leads to the conclusion that this algorithm has $O\left(\left|V_{N}\right| n\right)$ time complexity.

\subsection{Island Probability $-\operatorname{Pr}\left(H \stackrel{*}{\Rightarrow} \Sigma^{*} w_{i} \ldots w_{i+n} \Sigma^{*}\right)$}

The island probability consists of $\operatorname{Pr}\left(H \stackrel{*}{\Rightarrow} \Sigma^{*} w_{i} \ldots w_{i+n} \Sigma^{*}\right)$, the probability of all derivation trees with root in $H$ that generate arbitrary length sequences containing the subsequence $w_{i} \ldots w_{i+n}$.

Let

$$
\begin{aligned}
\mathrm{P}_{R}(H \rightarrow G) \stackrel{\text { def }}{=} & \sum_{\gamma} \operatorname{Pr}(H \rightarrow \gamma G) \quad, \gamma \in \Sigma^{*} \\
= & \text { probability of rewriting } H \text { by sequences with } \\
& \text { the nonterminal symbol } G \text { as suffix }
\end{aligned}
$$

One can write

$$
\begin{aligned}
& \operatorname{Pr}\left(\sigma \stackrel{*}{\Rightarrow} \Sigma^{*} w_{i} \ldots w_{i+n} \Sigma^{*}\right)=\sum_{G} \operatorname{Pr}(\sigma \rightarrow G) \operatorname{Pr}\left(G \stackrel{*}{\Rightarrow} \Sigma^{*} w_{i} \ldots w_{i+n} \Sigma^{*}\right) \\
& \operatorname{Pr}\left(H \stackrel{*}{\Rightarrow} \Sigma^{*} w_{i} \ldots w_{i+n} \Sigma^{*}\right) \quad, \quad H \neq \sigma \\
& =\sum_{G} \operatorname{P}_{R}(H \rightarrow G) \operatorname{Pr}\left(G \stackrel{*}{\Rightarrow} \Sigma^{*} w_{i} \ldots w_{i+n} \Sigma^{*}\right)+ \\
& \quad+\sum_{G} \sum_{j=1}^{n_{\text {min }}(H, G)} \operatorname{P}_{R}\left(H \rightarrow w_{i} \ldots w_{i+j-1} G\right) \operatorname{Pr}\left(G \stackrel{*}{\Rightarrow} w_{i+j} \ldots w_{i+n} \Sigma^{*}\right)+ \\
& \quad+\sum_{G} \operatorname{P}_{R}\left(H \rightarrow w_{i} \ldots w_{i+n} G\right) \underbrace{\operatorname{Pr}\left(G \stackrel{*}{\Rightarrow} \Sigma^{*}\right)}_{=1}+
\end{aligned}
$$




$$
\begin{aligned}
& +\sum_{G} \sum_{j, k} \operatorname{Pr}\left(H \rightarrow v_{1} \ldots v_{k} w_{i} \ldots w_{i+n} z_{1} \ldots z_{j} G\right) \underbrace{\operatorname{Pr}\left(G \stackrel{*}{\Rightarrow} \Sigma^{*}\right)}_{=1}+ \\
& +\sum_{G} \sum_{j, k} \operatorname{Pr}\left(H \rightarrow v_{1} \ldots v_{k} w_{i} \ldots w_{i+n} z_{1} \ldots z_{j}\right)
\end{aligned}
$$

For strings sufficiently long $\left(n>>_{G}^{\max }\left\{C_{T}(\gamma): G \rightarrow \gamma\right\}\right.$ ) the last three terms do not exist, therefore we will ignore them from now on.

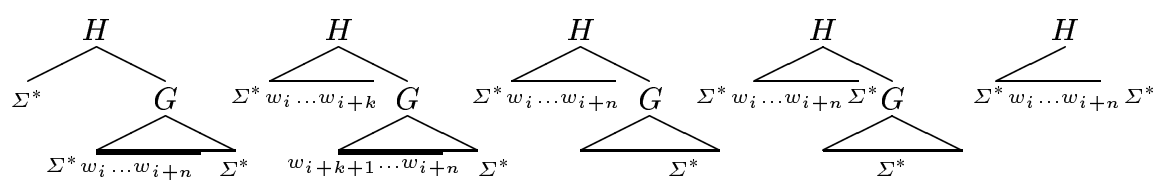

Fig. 4. Terms of the expression 14 .

$$
\begin{aligned}
& \operatorname{Pr}\left(H \stackrel{*}{\Rightarrow} \Sigma^{*} w_{i} \ldots w_{i+n} \Sigma^{*}\right) \quad, \quad H \neq \sigma \\
& =\sum_{G} \operatorname{P}_{R}(H \rightarrow G) \operatorname{Pr}\left(G \stackrel{*}{\Rightarrow} \Sigma^{*} w_{i} \ldots w_{i+n} \Sigma^{*}\right)+ \\
& \quad+\sum_{G} \sum_{j=1}^{n_{\min (H, G)}} \mathrm{P}_{R}\left(H \rightarrow w_{i} \ldots w_{i+j-1} G\right) \operatorname{Pr}\left(G \stackrel{*}{\Rightarrow} w_{i+j} \ldots w_{i+n} \Sigma^{*}\right)
\end{aligned}
$$

Recursively applying the above expression and after some manipulation (details can be found in [6]) one obtains:

$$
\begin{aligned}
& \operatorname{Pr}\left(H \stackrel{*}{\Rightarrow} \Sigma^{*} w_{i} \ldots w_{i+n} \Sigma^{*}\right) \quad, \quad H \neq \sigma \\
& =\sum_{A} Q_{R}(H \Rightarrow A) \sum_{G} \sum_{j \in \mathcal{N}^{+}} \mathrm{P}_{R}\left(A \rightarrow w_{i} \ldots w_{i+j-1} G\right) \operatorname{Pr}\left(G \stackrel{*}{\Rightarrow} w_{i+j} \ldots w_{i+n} \Sigma^{*}\right) \\
& \quad+\sum_{G} \sum_{j \in \mathcal{N}^{+}} \mathrm{P}_{R}\left(H \rightarrow w_{i} \ldots w_{i+j-1} G\right) \operatorname{Pr}\left(G \stackrel{*}{\Rightarrow} w_{i+j} \ldots w_{i+n} \Sigma^{*}\right)
\end{aligned}
$$

with

$$
\begin{aligned}
& Q_{R}(H \Rightarrow G)=\operatorname{Pr}\left(H \stackrel{*}{\Rightarrow} \Sigma^{*} G\right) \\
& =\mathrm{P}_{R}(H \rightarrow G)+ \\
& \quad+\sum_{A} \mathrm{P}_{R}(H \rightarrow A) \mathrm{P}_{R}(A \rightarrow G)+\sum_{A_{1} A_{2}} \mathrm{P}_{R}\left(H \rightarrow A_{1}\right) \mathrm{P}_{R}\left(A_{1} \rightarrow A_{2}\right) \times \\
& \quad \times \mathrm{P}_{R}\left(A_{2} \rightarrow G\right)+\ldots
\end{aligned}
$$

$\mathrm{Q}_{R}(H \Rightarrow G)$ obeys the equation

$$
\mathrm{Q}_{R}(H \Rightarrow G)=\sum_{A} \mathrm{P}_{R}(H \rightarrow A) \mathrm{Q}_{R}(A \Rightarrow G)+\mathrm{P}_{R}(H \rightarrow G)
$$


Defining the matrices

$$
\begin{aligned}
& \mathcal{P}_{R}[H, G]=\mathrm{P}_{R}(H \rightarrow G) \\
& \mathcal{Q}_{R}[H, G]=\mathrm{Q}_{R}(H \Rightarrow G)
\end{aligned}
$$

$\mathcal{Q}$ is given by $[6]$ :

$$
\mathcal{Q}_{R}=\mathcal{P}_{R}\left[I-\mathcal{P}_{R}\right]^{-1}
$$

The algorithm can thus be described as:

1. Off-line computation of $\mathcal{Q}_{R}=\mathcal{P}_{R}\left[I-\mathcal{P}_{R}\right]^{-1}$.

2. On-line computation of $\operatorname{Pr}\left(G \stackrel{*}{\Rightarrow} w_{n} \Sigma^{*}\right), \operatorname{Pr}\left(G \stackrel{*}{\Rightarrow} w_{n-1} w_{n} \Sigma^{*}\right), \ldots, \operatorname{Pr}(G \stackrel{*}{\Rightarrow}$ $\left.w_{2} \ldots w_{n} \Sigma^{*}\right)$ for all nonterminal symbols $G \in V_{N}-\{\sigma\}$ using the algorithm in section 3.3 .

3. For all nonterminal symbols $H \in V_{N}-\{\sigma\}$ compute

$$
\begin{aligned}
& \operatorname{Pr}\left(H \stackrel{*}{\Rightarrow} \Sigma^{*} w_{i} \ldots w_{i+n} \Sigma^{*}\right) \\
& =\sum_{A} Q_{R}(H \Rightarrow A) \sum_{G} \sum_{j \in \mathcal{N}^{+}} \mathrm{P}_{R}\left(A \rightarrow w_{i} \ldots w_{i+j-1} G\right) \times \\
& \quad \times \operatorname{Pr}\left(G \stackrel{*}{\Rightarrow} w_{i+j} \ldots w_{i+n} \Sigma^{*}\right)+ \\
& \quad+\sum_{G} \sum_{j \in \mathcal{N}^{+}} \mathrm{P}_{R}\left(H \rightarrow w_{i} \ldots w_{i+j-1} G\right) \operatorname{Pr}\left(G \stackrel{*}{\Rightarrow} w_{i+j} \ldots w_{i+n} \Sigma^{*}\right)
\end{aligned}
$$

4.

$$
\operatorname{Pr}\left(\sigma \stackrel{*}{\Rightarrow} \Sigma^{*} w_{i} \ldots w_{i+n} \Sigma^{*}\right)=\sum_{G} \operatorname{Pr}(\sigma \rightarrow G) \operatorname{Pr}\left(G \stackrel{*}{\Rightarrow} \Sigma^{*} w_{i} \ldots w_{i+n} \Sigma^{*}\right)
$$

The required on-line computations have the following time complexity:

$$
\begin{aligned}
& O(\underbrace{\left(\left|V_{N}\right|-1\right)(n-1) \alpha}_{\text {step } 2}+\underbrace{\left(\left|V_{N}\right|-1\right)\left|V_{N}\right| \beta}_{\text {step } 3}+\underbrace{\delta}_{\text {step } 4})= \\
& =\max \left(O\left(\left|V_{N}\right| n\right), O\left(\left|V_{N}\right|^{2}\right)\right.
\end{aligned}
$$

\subsection{Suffix Probability $-\operatorname{Pr}\left(H \stackrel{*}{\Rightarrow} \Sigma^{*} w_{i} \ldots w_{i+n}\right)$}

Let $\operatorname{Pr}\left(H \stackrel{*}{\Rightarrow} \Sigma^{*} w_{i} \ldots w_{i+n}\right)$ be the probability of all derivation trees with root in $H$ generating arbitrary length strings having $w_{i} \ldots w_{i+n}$ as a suffix. This probability can be derived as follows:

$$
\operatorname{Pr}\left(\sigma \stackrel{*}{\Rightarrow} \Sigma^{*} w_{i} \ldots w_{i+n}\right)=\sum_{G} \operatorname{Pr}(\sigma \rightarrow G) \operatorname{Pr}\left(G \stackrel{*}{\Rightarrow} \Sigma^{*} w_{i} \ldots w_{i+n}\right)
$$




$$
\begin{aligned}
& \operatorname{Pr}\left(H \stackrel{*}{\Rightarrow} \Sigma^{*} w_{i} \ldots w_{i+n}\right) \quad, \quad H \neq \sigma \\
& =\sum_{G} \mathrm{P}_{R}(H \rightarrow G) \operatorname{Pr}\left(G \stackrel{*}{\Rightarrow} \Sigma^{*} w_{i} \ldots w_{i+n}\right)+ \\
& \quad+\sum_{G} \sum_{j=1}^{n_{\text {min }(H, G)}} \mathrm{P}_{R}\left(H \rightarrow w_{i} \ldots w_{i+j-1} G\right) \operatorname{Pr}\left(G \stackrel{*}{\Rightarrow} w_{i+j} \ldots w_{i+n}\right)+ \\
& \quad+\sum_{G} \mathrm{P}_{R}\left(H \rightarrow w_{i} \ldots w_{i+n}\right)
\end{aligned}
$$

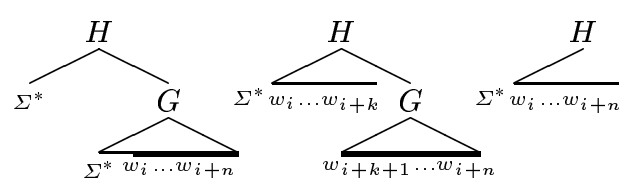

Fig. 5. Terms of the expression 23 .

For strings sufficiently long $\left(n>>_{G}^{\max }\left\{C_{T}(\gamma): G \rightarrow \gamma\right\}\right.$ ) the third term does not exist, so it will be ignored henceforth. Recursively applying the resulting expression to the second part of the first term one obtains:

$$
\begin{aligned}
\operatorname{Pr} & \left.H \stackrel{*}{\Rightarrow} \Sigma^{*} w_{i} \ldots w_{i+n}\right) \quad, \quad H \neq \sigma \\
= & \sum_{A_{1}} \sum_{j \in \mathcal{N}^{+}} \mathrm{P}_{R}\left(H \rightarrow w_{i} \ldots w_{i+j-1} A_{1}\right) \operatorname{Pr}\left(A_{1} \stackrel{*}{\Rightarrow} w_{i+j} \ldots w_{i+n}\right)+ \\
& +\sum_{A_{1}, A_{2}} \sum_{j \in \mathcal{N}^{+}} \mathrm{P}_{R}\left(H \rightarrow A_{1}\right) \mathrm{P}_{R}\left(A_{1} \rightarrow w_{i} \ldots w_{i+j-1} A_{2}\right) . \\
& . \operatorname{Pr}\left(A_{2} \Rightarrow w_{i+j} \ldots w_{i+n}\right)+ \\
& +\ldots+ \\
& +\sum_{A_{1}, \ldots, A_{k}} \mathrm{P}_{R}\left(H \rightarrow A_{1}\right) \mathrm{P}_{R}\left(A_{1} \rightarrow A_{2}\right) \ldots \\
& \ldots . \mathrm{P}_{R}\left(A_{k-1} \rightarrow w_{i} \ldots w_{i+j-1} A_{k}\right) \operatorname{Pr}\left(A_{k} \stackrel{*}{\Rightarrow} w_{i+j} \ldots w_{i+n}\right)+\ldots \\
= & \sum_{A} Q_{R}(H \Rightarrow A) \sum_{G} \sum_{j \in \mathcal{N}^{+}} \mathrm{P}_{R}\left(A \rightarrow w_{i} \ldots w_{i+j-1} G\right) \operatorname{Pr}\left(G \stackrel{*}{\Rightarrow} w_{i+j} \ldots w_{i+n}\right)+ \\
& +\sum_{G} \sum_{j \in \mathcal{N}^{+}} \mathrm{P}_{R}\left(H \rightarrow w_{i} \ldots w_{i+j-1} G\right) \operatorname{Pr}\left(G \stackrel{*}{\Rightarrow} w_{i+j} \ldots w_{i+n}\right)
\end{aligned}
$$

The algorithm is then:

1. Off-line computation of $\mathcal{Q}_{R}=\mathcal{P}_{R}\left[I-\mathcal{P}_{R}\right]^{-1}$. 
2. On-line computation of $\operatorname{Pr}\left(G \stackrel{*}{\Rightarrow} w_{n}\right), \operatorname{Pr}\left(G \stackrel{*}{\Rightarrow} w_{n-1} w_{n}\right), \ldots, \operatorname{Pr}(G \stackrel{*}{\Rightarrow}$ $\left.w_{2} \ldots w_{n}\right)$ for all non-terminal symbols $G \in V_{N}-\{\sigma\}$ using the algorithm in section 3.1.

3. For all non-terminal symbols $H \in V_{N}-\{\sigma\}$ compute

$$
\begin{aligned}
& \operatorname{Pr}\left(H \stackrel{*}{\Rightarrow} \Sigma^{*} w_{i} \ldots w_{i+n}\right) \\
& =\sum_{A} Q_{R}(H \Rightarrow A) \sum_{G} \sum_{j \in \mathcal{N}^{+}} \mathrm{P}_{R}\left(A \rightarrow w_{i} \ldots w_{i+j-1} G\right) \operatorname{Pr}\left(G \stackrel{*}{\Rightarrow} w_{i+j} \ldots w_{i+n}\right) \\
& \quad+\sum_{G} \sum_{j \in \mathcal{N}^{+}} \mathrm{P}_{R}\left(H \rightarrow w_{i} \ldots w_{i+j-1} G\right) \operatorname{Pr}\left(G \stackrel{*}{\Rightarrow} w_{i+j} \ldots w_{i+n}\right)
\end{aligned}
$$

4.

$$
\operatorname{Pr}\left(\sigma \stackrel{*}{\Rightarrow} \Sigma^{*} w_{i} \ldots w_{i+n}\right)=\sum_{G} \operatorname{Pr}(\sigma \rightarrow G) \operatorname{Pr}\left(G \stackrel{*}{\Rightarrow} \Sigma^{*} w_{i} \ldots w_{i+n}\right)
$$

On-line computations have the time complexity:

$$
O(\underbrace{\left(\left|V_{N}\right|-1\right)(n-1) \alpha}_{\text {step } 2}+\underbrace{\left(\left|V_{N}\right|-1\right) \beta}_{\text {step } 3}+\underbrace{\delta}_{\text {step } 4})=O\left(\left|V_{N}\right| n\right)
$$

\section{Conclusions}

This paper described several algorithms for the computation of substring probabilities according to grammars written in the form

$$
\sigma \rightarrow F_{i} \quad, \quad F_{i} \rightarrow \alpha \quad, \quad F_{i} \rightarrow \alpha F_{j} \quad, \quad \alpha \in \Sigma^{*} \quad, \quad \sigma, F_{i}, F_{j} \in V_{N}
$$

Table 1 summarizes the probabilities considered here, and the order of complexity of the associated algorithms.

\begin{tabular}{|l|l|c|}
\hline & Expression & $\begin{array}{c}\text { Algorithm } \\
\text { time Complexity }\end{array}$ \\
\hline Fixed length & $\operatorname{Pr}\left(H \stackrel{*}{\Rightarrow} w_{1} \ldots w_{n}\right)$ & $O\left(\left|V_{N}\right| n\right)$ \\
strings & $\operatorname{Pm}_{\mathrm{m}}\left(H \stackrel{*}{\Rightarrow} w_{1} \ldots w_{n}\right)$ & $O\left(\left|V_{N}\right| n\right)$ \\
\hline Arbitrary & $\operatorname{Pr}\left(H \stackrel{*}{\Rightarrow} w_{1} \ldots w_{n} \Sigma^{*}\right)$ & $O\left(\left|V_{N}\right| n\right)$ \\
length & $\operatorname{Pr}\left(H \stackrel{*}{\Rightarrow} \Sigma^{*} w_{1} \ldots w_{n} \Sigma^{*}\right)$ & $\max \left(O\left(\left|V_{N}\right| n\right), O\left(\left|V_{N}\right|^{2}\right)\right.$ \\
strings & $\operatorname{Pr}\left(H \stackrel{*}{\Rightarrow} \Sigma^{*} w_{1} \ldots w_{n}\right)$ & $O\left(\left|V_{N}\right| n\right)$ \\
\hline
\end{tabular}

Table 1. Summary of proposed algorithms for the computation of sub-string probabilities. 
More general algorithms for the computation of sub-string probabilities according to stochastic context-free grammars, written in Chomsky Normal Form, can be found in [1-3]. However, the later have $O\left(n^{3}\right)$ time complexity [2]. The herein proposed algorithms, exhibiting linear time complexity in string's length, represent a computationally appealing alternative to be used whenever the application at hand can adequately be modeled by the types of grammars described above. Examples of application of the algorithms described in this paper can be found in $[5,6,8,9]$.

\section{References}

1. F. Jelinek, J. D. Lafferty and R. L. Mercer. Basic Methods of Probabilistic Context Free Grammars. In Speech Recognition and Understanding. Recent Advances, pages 345-360. Springer-Verlag, 1992.

2. A. Corazza, R. De Mori, R. Gretter, and G. Satta. Computation of Probabilities for an Island-Driven Parser. IEEE Trans. Pattern Anal. Machine Intell., vol. 13, No. 9, pages 936-949, 1991.

3. A. Corazza, R. De Mori, and G. Satta. Computation of Upper-Bounds for Stochastic Context-Free Languages. In proceedings AAAI-92, pages 344-349, 1992.

4. A. Corazza, R. De Mori, R. Gretter, and G. Satta. Some Recent results on Stochastic Language Modelling. In Advances in Structural and Syntactic Pattern Recognition, World-Scientific, pages 163-183, 1992.

5. A. L. N. Fred, A. C. Rosa, and J. M. N. Leitão. Predicting REM in sleep EEG using a structural approach. In E. S. Gelsema and L. N. Kanal, editors, Pattern Recognition in Practice IV, pages 107 - 117. Elsevier Science Publishers, 1994.

6. A. L. N. Fred, Structural Pattern Recognition: Applications in Automatic Sleep Analysis, PhD Thesis, Technical University of Lisbon, 1994.

7. K. S. Fu and T. L. Booth. Grammatical inference: Introduction and survey - part I and II. IEEE Trans. Pattern Anal. Machine Intell., PAMI-8:343-359, May 1986.

8. Ana L. N. Fred and T. Paiva. Sleep Dynamics and Sleep Disorders: a Syntactic Approach to Hypnogram Classification. Em 10th Nordic-Baltic Conference on Biomedical Engineering and 1st International Conference on Bioelectromagnetism, pp 395396, Tampere, Finlândia Junho, 1996.

9. A. L. N. Fred, J. S. Marques, P. M. Jorge, Hidden Markov Models vs Syntactic Modeling in Object Recognition, Proc. Intl. Conference on Image Processing,ICIP'97, 1997. 